Research Article

\title{
Development of Combinational Circuits by Encoding on the Basis of Developmental Biology
}

\author{
S. B. Sivasubramaniyan $\left(\mathbb{D},{ }^{1}\right.$ R. Seshasayanan, ${ }^{2}$ and N. Ramadass ${ }^{2}$ \\ ${ }^{1}$ Department of Electrical and Electronics Engineering, Meenakshi Sundararajan Engineering College, Anna University, \\ Chennai, India \\ ${ }^{2}$ Department of Electronics and Communication Engineering, College of Engineering, Guindy, Anna University, Chennai, India
}

Correspondence should be addressed to S. B. Sivasubramaniyan; sbsquest@gmail.com

Received 12 November 2019; Accepted 13 February 2020; Published 1 July 2020

Academic Editor: Sandhya Samarasinghe

Copyright (c) 2020 S. B. Sivasubramaniyan et al. This is an open access article distributed under the Creative Commons Attribution License, which permits unrestricted use, distribution, and reproduction in any medium, provided the original work is properly cited.

\begin{abstract}
The present work visualizes the evolution of primitive digital circuits as a development problem. The development of the digital circuit is implemented similar to the development of a human embryo from a single cell to the complete organism. The constituent parts making up a primitive digital circuit are encoded into binary strings. Each binary string is viewed as a cell, and several such cells are allowed to adhere and multiply before culminating into a developed organism. The binary string of the cell is further mapped to a particular attribute which defines the constituent of the complete digital circuit implemented. The present work illustrates the development of a 4 -input combinational digital circuit. The development of 2-input majority function is illustrated, and the results are shown for the 2-input Ex-OR gate, 2-input majority function with 4 input variables, and a 2-to-1 multiplexer circuit. The development of the digital circuit resembles the development of an embryo in a living organism.
\end{abstract}

\section{Introduction}

The biological world has demonstrated that the fittest organism survives over time. A change in the organism's genome results in initial changes in the phenotype. These changes are manifested in the offspring over time. We refer to this process as evolution. The change occurring in the organism's genotype is either random or forced by the environment. Understanding and mimicking nature has led to a whole new field of evolutionary algorithms, whose primary objectives are search and optimization of multivariable problems. Genetic algorithm (GA), evolutionary strategies (ES), and evolutionary programming (EP) are alternative strategies followed by different groups of researchers working on a range of diverse problems.

The present work is based on the premise that development is the key to evolution. The adaptability of the any organism rests on the flexibility of the organism to the changes within and outside it. The flexibility and the eventual adaptability would be meaningful only when the organizational framework of the organism is intact. Thus, development is seen as the key for evolution of a species. The proposed work is an attempt to emulate the adaptability of the biological organism to digital hardware.

Biological species undergoes a highly deterministic developmental cycle. The embryonic development of any species starts with a single cellular organism. The single cell undergoes process consisting of more than one developmental stage, crafted naturally, to become a multicellular organism. The proposed work is aimed at creating a developmental cycle for the design of digital hardware. Digital hardware is represented by a Boolean expression. A Boolean expression consisting of few gates can be represented either as Sum of Product or as Product of Sum expressions. The proposed work tried to find out whether the Boolean expression can be developed using primitives mimicking the developmental cycle of a biological organism.

The present work is also motivated by attempts including Mandelbrot sets, in which Mandelbrot developed patterns using a simple equation. Lindenmayer systems, commonly 
referred as L-systems, relied on step-by-step procedures to develop complex and symmetric patterns from single primitives.

The present work proposes a developmental algorithm, similar in line with the embryonic development of a biological species, for the electronic circuit. In the process, distinct encoding of each component of a circuit by a binary string is carried out. Encoding of each of the component and its association with other components of the circuit is carried out which resembled the DNA encoding of the biological species. The binary representation also paved the necessary edge to include mutation by means of flipping of bits. The genome of a circuit is considered to be specific to the functionality of the circuit.

The proposed work aims at the development of the electronic circuit as an embryo develops in a biological world. This is carried out by binary encoding of each of the components and then designing an algorithm for the development of the circuit. The proposed work would be an ideal platform to the design of fault tolerant systems and selfhealing circuits.

\section{Literature Survey}

There have been attempts carried out to determine the adaptability of various evolutionary methods in optimization of various problems [1]. Among the engineering problems successfully adopted for the practical hardware design, the most important are the design of an antenna [2], table [3], rotor blade design [4], and physical properties of the silicon substrate [5]. It may be mentioned in passing that social behavior of birds [6], lion [7-9], and human beings [10] also has been formulated as an optimization problem.

The development of a digital circuit with fundamental gates starts with a truth table of the circuit. For $n$ variables, it requires $2 n$ combinations to be checked for determining a Boolean expression. This problem is referred to as the satisfiability (SAT) problem. The time required for a Boolean SAT problem increases exponentially with increase in the number of variables. Perdrycz et al. handled the challenges in applying evolutionary computation (EC) for a Boolean SAT problem by converting the Boolean problem to a continuous domain [11]. De Jong and Spears handled the same by converting Boolean variables to floating point numbers [12]. Slowik and Bialko in [13] presented a comprehensive survey on the application of EAs to digital circuit design.

The idea of evolving optimized circuits by encoding the components and the interconnections among them has been demonstrated in [14]. An attempt to apply genetic programming to evolve a fit computer program is presented by Koza in [15]. Coello [16] employed the genetic algorithm to design adders and multipliers. In [17], Miller et al. proposed genetic algorithm-based combinational circuit design which was modeled on a FPGA. These efforts led to the design and development of digital circuits built on an array of gates controlled by predetermined criteria $[18,19]$. A modified model, called the development model having two layers, a protein layer and an architecture layer, was proposed by Gordon and Bentley in [20]. The present work aims at developing a digital circuit without a predefined array. The later sections discuss the same in detail. Section 2 deals with the system design using artificial cells. The proposed algorithm is given in Section 3. A combinational circuit example is discussed in Section 4. Section 5 discusses the results of the algorithm.

\section{System Design with Artificial Cells}

3.1. Development of Combinational Circuits. This paper proposes a model for the development of a digital circuit. Various components making up the circuit are visualized as a biological cell making up an organ. Each component, with its associated input pattern, is encoded with a unique binary string. For a 7-level digital circuit, the length of the encoded binary string for a single cell is defined by an empirical relation given in the following equation:

$$
\text { length of the genome }=\log _{2} i+2 i+13 \ldots \ldots \ldots \text {, }
$$

where " $i$ " is the number of inputs to the logic circuit. The genetic strip is referred to as the genome of the cell, and the combination of several cells makes up the eventual digital circuit. The visualized genetic strip accommodates designs consisting of basic logic gates, derived gates, and universal gates along with logic circuits. The unused genes merely serve the purpose of filling up spaces as of now.

A Sum of Product (SoP) expression is taken for the illustration. The truth table of the problem is taken as an input. This makes the problem a Boolean satisfiability (SAT) problem. The time required to attain the solution increases with the number of variables being taken. The major challenge to the SAT problem is the definition of the fitness function which is problem-dependent. The natural way is defining the fitness function with logical " 1 " entries in the truth table. However, it is understood that logical " 1 " is not the complete solution of the function, rather one of the possibilities of the complete solution, as all the functions making up the truth table are equally competitive [11]. This is justified as the development problem overcomes the scalability issue associated with the digital circuit evolution [20].

The proposed idea contains problem-specific (speciesspecific) cycles and each cycle comprising one or many phases. The cycles and phases are modeled to follow the steps presented in the following.

3.1.1. Birth of Zygote. Artificial cell division starts with an initial randomly chosen single cell made up of the binary string. The single cell represents the zygote in our artificial cellular development. Naturally, one of the functions in the truth table having a logic " 1 " value will be the zygote. The zygote is randomly chosen from all the possibilities posed by the truth table of the function. If the zygote fails the fitness test, it eventually 'dies,' and the new zygote is produced. The birth of zygote is referred as phase I of the artificial model.

3.1.2. Cell Division: SAT Problem. In phase II, the single cell divides itself into two. The two daughter cells will have a single-bit change from the parent. Again, each of the 
daughter cells divides into two. The single-bit change will take place in one of the frames denoting the input type. Cells divide till the "telomere index" becomes zero. The telomere index is defined in the later sections.

3.1.3. Single-Bit Change: Concentration Gradient and the Inducing Factor. In natural cells, the concentration gradient and the inducing factor start from one point and gradually reach some other point of the cell during gastrulation. The single-bit change resembles the concentration gradient in the presence of the inducing factor in the biological world. If the bit string is "0100," the daughter cells produced would be "0101" and "0111." This is after considering that the bit change is taken from right to left. If it is taken from left to right, it would be "1100" and "1000." Figure 1 shows the production of daughter cells from parent cells. The natural processes stated above-cell division, blastulation, and gastrulation-are assumed to take place simultaneously in our artificial model. We refer this as phase II. The single-bit changes in the strings are checked for their fitness. If the cells are not fit, the telomere index is unaltered. If the cells are fit and there is no repetition, the telomere index is decremented by one. The fitness test carried out is the genotypic fitness.

At the end of gastrulation, morphogenesis takes place. Morphogenesis defines the structure to the organism. Several types of morphogenetic processes are described [21]. The morphogenetic processes are species-specific and organspecific in a particular species. Similar in line with that, the proposed morphogenetic process is designed to follow condensation in which mesenchymal cells undergo local cell division and consequent cell adhesion. Slack describes that the cells having similar cadherins adhere than the cells with dissimilar cadherins [21].

3.1.4. Local Cell Division: Cell Replication. This is similar to the local cell division in the biological world. Cells which undergo adhesion are defined by a factor denoted as "specificity factor" or simply "s-factor." Adhesion is defined for the cell pair which has a high specificity factor. If the two cells have the exact genetic material, they fuse together to form one cell, so s-factor of identical cells is discarded. If two cells have only one-bit (gene) change between them, then the cells are said to have more s-factor. Gate-dominated adhesions are possible only for cells having one-bit difference among them. Gate-dominated adhesions are referred as "gadherins." Specificity factor (s-factor) also plays a role in local cell division or cell replication. A replication index or " $r$-index" (or simply " $r$ ") is defined for the number of identical copies the cell makes up during local cell division. $s$-factor and $r$-index work sequentially. Each cell having high "s-factor" is given $(r-1)$ replications in the local cell division.

3.1.5. Cell Adhesion and Specificity: Laws of Complementarity. Before any cell adhesion is possible, cells are tested for their specificity with each other cell. A " $n$-" cell system will have $n \mathrm{C}_{2}$ number of combinations possible. Say, for example, a 4cell system will have 6 possible combinations of two cells taken at a time (a $4 \mathrm{C}_{2}$ problem). All the resultant combinations are tested for their specificity. Cells with high sfactor now are chosen to take part in gate-dominated adhesions after cells locally make $(r-1)$ copies of it.

Each copy of the cell undergoes adhesion with the other types of the cell. This takes into account the Boolean function, $X+X^{\prime}=1$ and $\mathrm{X} \cdot \mathrm{X}^{\prime}=0$. The cell adhesion produces distinct cells after the fusion; the cells which have high specificity factor alone produce the fused products. The cells which do not find a proper ally to adhere stay in the system unscathed as shown in Figure 2.

There are two types of cell fusion defined in the artificial world-AND fusion and OR fusion. The SoP realization, obviously, follows AND fusion in cycle I of development. The prerequisite for two cells to undergo fusion (adhesion) is that the two cells must have only one-bit difference between them. If the prerequisite is met, the cells undergo adhesion and produce a single distinct cell with a logic ' 0 ' in the allele which had the single-bit difference. OR fusion makes a logic "1" in the corresponding allele. We refer this as gateDominated adhesion or simply, gadherins. AND fusion is referred as gadherin type- 1 or GT-1 adhesion and OR, GT-2 adhesion.

\subsubsection{Differential Strength of Adhesion: Consensus Theorem.} The strength of adhesion of each cell with the other cell is quantified to minimize the logic function being implemented. The differential strength of adhesion also determines the stay of the cell in the system. Each cell adhesion forms "1" (not a logical "1"). If the number of cells forming "1" is two in number, the two cells contribute equally to the adhesion -0.5 each, 0.25 each if there are 4 cells, and so on. If the same cell also forms the adhesion product with another cell, the cell further loses half of its strength-the initial 0.5 becoming 0.25 , and so on. This makes the original tally of " 1 " to reduce.

The reduction is permitted as long as the strength is not less than the individual contribution; in that case, the particular cell adhesion is ruled out. This takes into account the consensus theorem of the Boolean algebra. Figure 3 shows a 3-input function. Table 1 explains the differential strength of adhesion. The initial values are the values each cell possesses and final is the value each cell acquires at the end of all possible adhesions. When the cells adhere, the individual strengths of the cells account for the group.

Table 2 indicates the removal of weaker cells from the group. Table 2 signifies the phenotype fitness.

Phases I to III form cycle I in the proposed model. Cycle II follows cycle I based on the constraints given in the problem in hand. The presence of further cycles also depends on the problem.

3.2. Fitness Function. Inputs are fed as the constraint to be satisfied for the problem. The function that produces logic "1" is checked with all the combinations of the given set of inputs. These combinations are individually referred to as 


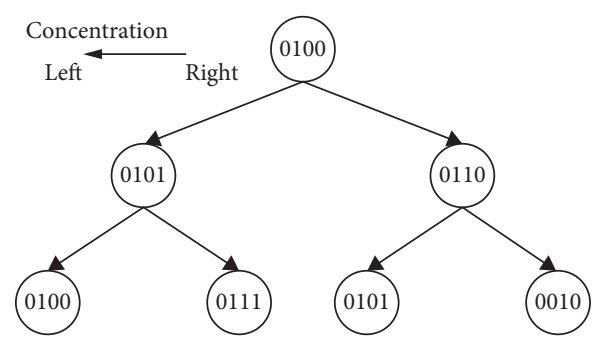

(a)

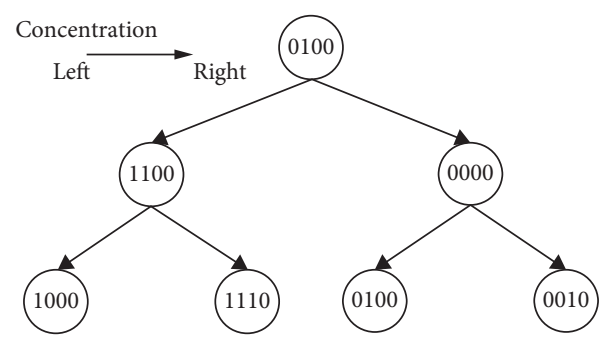

(b)

Figure 1: Parent and daughter cells differing by one-bit change.
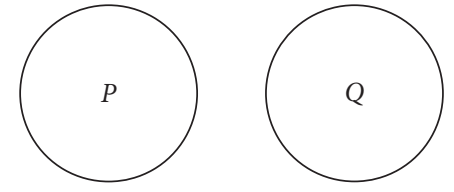

(a)

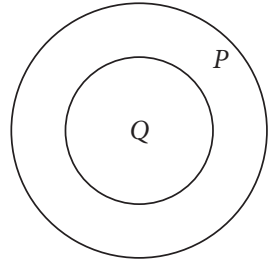

(b)
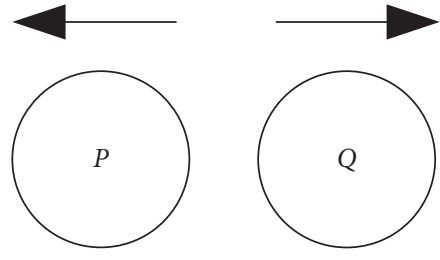

(c)

Figure 2: Two cells adhere together if the s-factor is high or move apart if the s-factor is low. (a) Two cells. (b) Adhesion of two cells (s-factor being high). (c) Two cells move apart (s-factor being low).

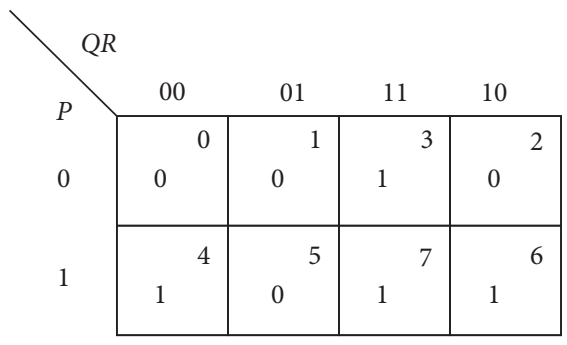

(a)

FIGURE 3: Grouping of 3-input function.

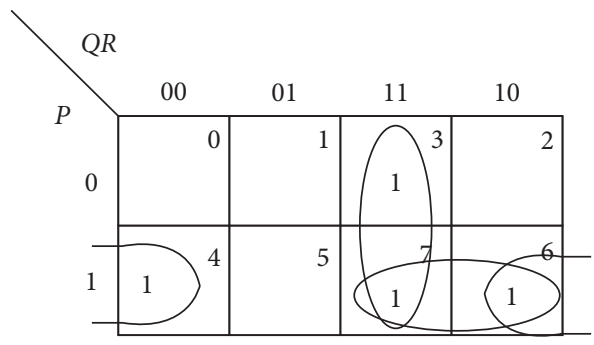

(b)
TABLE 1: Differential strengths of adhesion for the map shown in Figure 3.

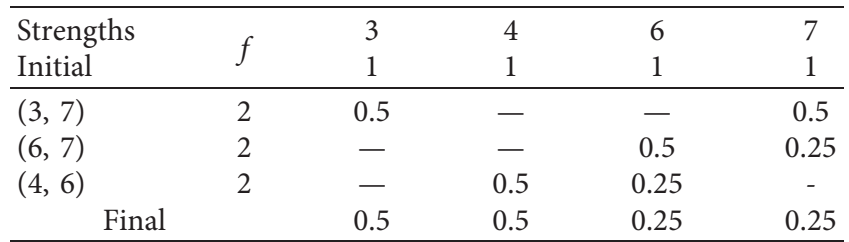

parts of the circuit to be formed. The fitness function is problem specific and more importantly, each cycle could well be assumed be have different fitness function either for components or for the nature of the inputs.

3.3. Illustration of the Genome. The circuit consists of various parts (or cells). Each cell is represented by a binary string referred as the genetic strip, genome, or chromosome. Each genetic strip is decoded into 5 frames. The length of each genetic strip or chromosome is determined using equation
TABLE 2: Removal of weaker cells.

\begin{tabular}{lccc}
\hline Adhered cell & Combined strength & Total & Status \\
\hline$(3,7)$ & $0.5+0.25$ & 0.75 & Selected \\
$(6,7)$ & $0.25+0.25$ & 0.50 & Discarded \\
$(4,6)$ & $0.5+0.25$ & 0.75 & Selected \\
\hline
\end{tabular}

(1). Frames are named as A, B, C, D, and E. Each genetic strip (chromosome) is unique for a particular cell during its birth. Upon development, the chromosome of a particular cell evolves and during the process, becomes robust. The cell achieves robustness by adhesion with the other cell. The number of bits or genes of the chromosome remains the same before and after the adhesion. The change gets affected only in the content of the chromosome, only at the particular allele, where the fusion takes place. The development starts with a single cell. The single cell is allowed to undergo the fitness test. After testing for fitness, the cell divides producing two daughter cells which in turn produce 4 daughter cells, and so on. 
TABle 3: Encoding of frames A and B.

\begin{tabular}{lcc}
\hline String & Frame A (input level) & Frame B (circuit level) \\
\hline 001 & Level I & Level I \\
011 & Level 2 & Level 2 \\
010 & Level 3 & Level 3 \\
110 & Level 4 & Level 4 \\
111 & Level 5 & Level 5 \\
101 & Level 6 & Level 6 \\
100 & Level 7 & Level 7 \\
\hline
\end{tabular}

A genetic strip or a chromosome with five frames is shown in the following. There is a start bit " 1 " before frame A and a stop bit " 1 " after frame E. The start and stop bits in the genetic strip represent the telomeres in the biological environment [21]. However, this electronic counterpart differs in two respects. The natural telomeres have repeated sequences of nucleotides, whereas in the proposed work, it is taken to be a single bit at the first and the last position of the genetic strip. The second difference is that the natural telomeres tend to lose few nucleotides during every time the cell divides, whereas in the defined telomere, it is taken care by the telomere index. The telomere index gets decremented each time the cell divides. By the time the telomeres fully disappear, the cells stop dividing. The artificial telomeres mere serve the purpose of start and stop bits of the genetic strip.

\begin{tabular}{|l|l|l|l|l|l|l|}
\hline Start & A & B & C & D & E & Stop \\
\hline
\end{tabular}

Each frame denotes an attribute of the chromosome. The details are shown in the following.

\begin{tabular}{|l|c|c|c|c|c|c|}
\hline Start & $\mathrm{A}$ & $\mathrm{B}$ & $\mathrm{C}$ & $\mathrm{D}$ & $\mathrm{E}$ & Stop \\
\hline Telomere & $\begin{array}{c}\text { Input } \\
\text { level }\end{array}$ & $\begin{array}{c}\text { Circuit } \\
\text { level }\end{array}$ & $\begin{array}{c}\text { Inputs \& } \\
\text { groups }\end{array}$ & $\begin{array}{c}\text { Type of } \\
\text { inputs }\end{array}$ & Component & Telomere \\
\hline
\end{tabular}

Equation (1) governs the size of the genome. For a 4-input and 7-level circuits, the size of the genome is as shown in the following. Frames $\mathrm{C}$ and $\mathrm{D}$ depend on the number of inputs as shown.

\begin{tabular}{|l|l|l|l|l|l|l|}
\hline 1 bit & 3 bit & 3 bit & 6 bit & 4 bit & 5 bit & 1 bit \\
\hline
\end{tabular}

The idea and the roles played by frames $\mathrm{A}$ and $\mathrm{B}$ are presented in Table 3.

Frame A in the strip indicates the level from which the input is applied to the circuit. Frame B represents the level of the circuit being considered. Table 4 explains the encodings of frames C and D. Frame C is made up of two smaller frames-c1 and $\mathrm{c} 2$. For a 4-input system considered, $\mathrm{c} 1$ has 2 bits, and c2 has 4 bits. Frame D represents whether the considered inputs are taken straight or complemented.

Table 5 denotes the encodings of frame E. Frame E has 5 bits with first 2 bits representing the component index, and the last 3 bits represent the type of the component.
Also, frames $\mathrm{C}$ and $\mathrm{D}$ undergo layered mutation. It is defined as one wherein a bit-level (gene) change in one frame affects the other. The change in frame D affects a corresponding change in frame $\mathrm{C}$. The corresponding changes in frames $C$ and $D$ are checked for a specific combination of bits (genes) in frame A, B, and E.

Once this is checked and deemed fit, the other set of genes is set in frames A, B, and E, and frames C and D are allowed to change. Layered mutations get affected in all the variations that happen in frames $C$ and D.

A chromosome given as 10010011111101011 represents a 2 -input AND gate. It is decoded as shown in the table which is given in the following.

\begin{tabular}{|l|c|c|c|c|c|c|}
\hline 1 & 001 & 001 & $1-11$ & 11 & 01011 & 1 \\
\hline Start & $\mathrm{A}$ & $\mathrm{B}$ & $\mathrm{C}$ & $\mathrm{D}$ & $\mathrm{E}$ & Stop \\
\hline & $\begin{array}{c}\text { Input } \\
\text { level }\end{array}$ & $\begin{array}{c}\text { Circuit } \\
\text { level }\end{array}$ & $\begin{array}{c}\text { Inputs \& } \\
\text { groups }\end{array}$ & $\begin{array}{c}\text { Input } \\
\text { type }\end{array}$ & Component & \\
\hline 1 bit & 3 bit & 3 bit & 3 bit & 2 bit & 5 bit & 1 \\
\hline
\end{tabular}

The chromosome has 18 genes arranged in 5 frames. Frame A has 001. This refers to the primary inputs which are fed to the prescribed gate (to be decoded with frame E). Frame B refers to level I of the circuit. Frame $C$ has two subframes-c1 and $c 2$. " 1 " in $\mathrm{c} 1$ refers that the number of inputs is 2 (say, $\mathrm{P}$ and $\mathrm{Q}$ ), and " 11 " in $\mathrm{c} 2$ denotes that both inputs are considered. Frame D which is decoded for the type (whether considered straight or complemented) of inputs denotes that the 2 inputs (P and $\mathrm{Q}$ ) are taken straight without complementing. Frame E has 5 bits. The first 2 bits are 01 which refers to Index 1 of the component. Component Index 1 refers to the fundamental gates-basic (AND, OR, and NOT), universal (NAND and NOR), and derived (Ex-OR and Ex-NOR). Frame $\mathrm{E}$ in the given genetic strip refers to AND gate (011). There is 1 bit each to signify the start and stop of the genetic strip. Overall, the chromosome referred by 10010011111101011 is decoded into $P \cdot Q$, or in other words, input $P$ and input $Q$ are fed to 2input AND gate.

With frame E as (01010) instead of (01011) would make the component to be an OR gate. This makes the function realized into $P+Q$. If the change is affected in frame $D$ with 01 in the original genome, the function realized is $P \cdot Q^{\prime}$ instead of $P \cdot Q$.

3.4. The Frame Swing. As stated already, each frame in the genetic strip has different fitness functions. The fitness check of the individual frames is carried out at different 
TABle 4: Encoding of frames $\mathrm{C}$ andD.

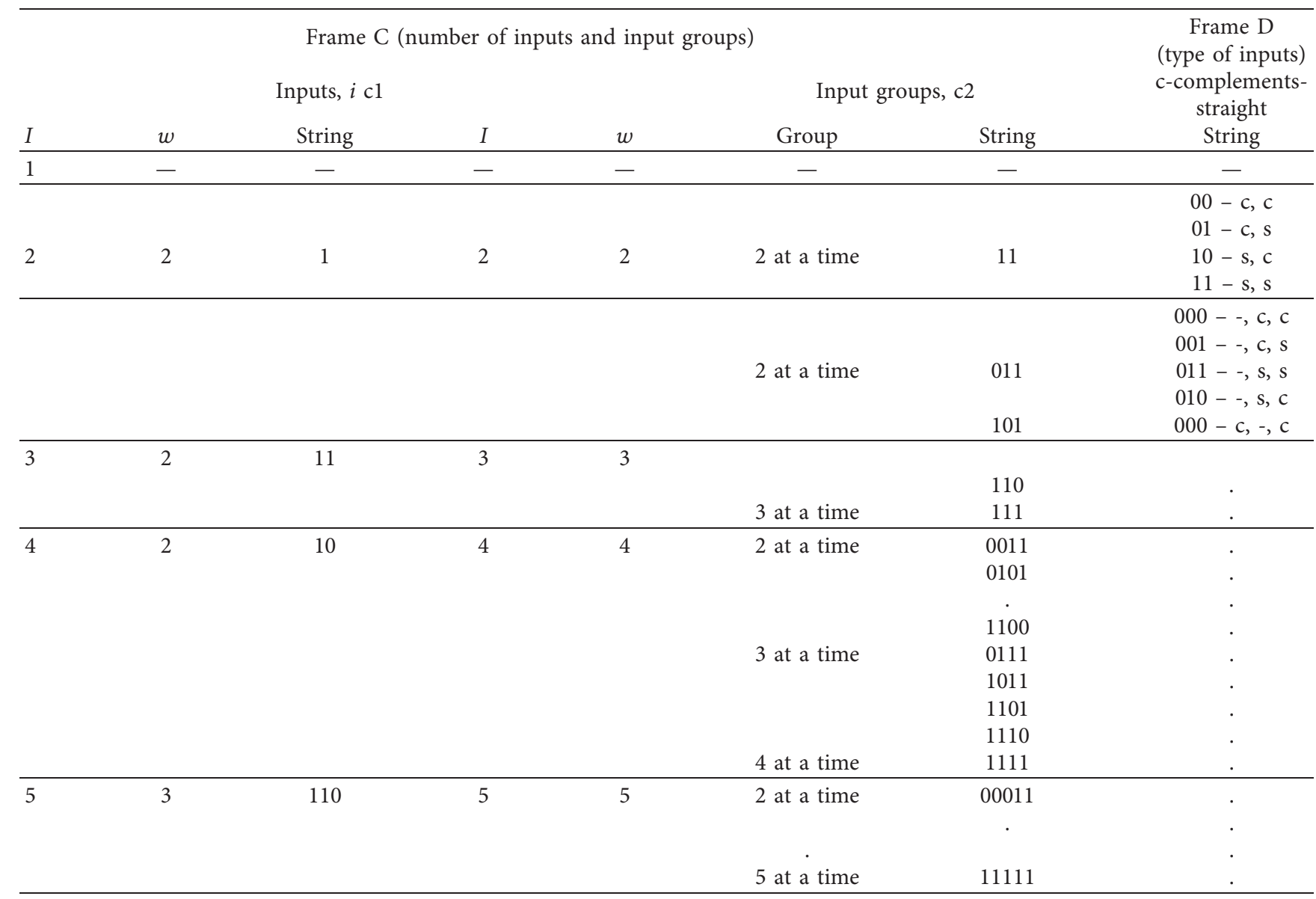

TABLE 5: Encoding of frame E.

\begin{tabular}{|c|c|c|}
\hline \multicolumn{3}{|c|}{ Frame E (components) } \\
\hline Index (encoding) & Index (decoded) & String \\
\hline 00 & - & - \\
\hline \multirow{8}{*}{01 (gates) } & Not defined & 000 \\
\hline & Ex-OR & 001 \\
\hline & AND & 011 \\
\hline & OR & 010 \\
\hline & Ex-NOR & 110 \\
\hline & Buffer & 111 \\
\hline & NOR & 101 \\
\hline & NAND & 100 \\
\hline \multirow{8}{*}{11 (circuits) } & Mux & 000 \\
\hline & Demux & 001 \\
\hline & Decoder & 011 \\
\hline & Encoder & 010 \\
\hline & . & 110 \\
\hline & . & 111 \\
\hline & . & 101 \\
\hline & . & 100 \\
\hline 10 & Empty & Empty \\
\hline
\end{tabular}

scales. The string change in frames A, B, and E occurs in parallel. Frame $\mathrm{C}$ undergoes random variations and completes its full set for a single variation with frames A,
B, and E. Frame D undergoes all possible changes for each string change in frame $\mathrm{C}$. We refer this as the frame swing (Algorithm 1). 


\subsection{The Proposed Algorithm}

\{

\{

Cycle I:

Defines the input level and the circuit level

Defines the number and nature of the inputs

Defines the type of the component

\{

Phase 1: Fertilization

Birth of a single cell zygote

\}

\{

Phase II: Cell Division: Embryogenesis-Genotypic fitness

Each cell divides itself into two.

Two cells divide into four. Four become eight, eight into sixteen, and so on.

Produces fit cells equaling the number of cells initially needed to make up the circuit.

\}

\{

Phase III: Cell Adhesion: Morphogenesis:

\{

Local Cell Division:

Cells which have high specificity with other cells replicate.

\}

\{

Gate Dominated Adhesions: Gadherins:

Replicated cells undergo gate-based adhesions with other cells.

Adhesions continue till distinct cells are produced.

\}

Differential Strength of Adhesions:

Each cell adheres differently to each of the other cells

\{

Determination of number of 1 s forming each group in " $C$ ":

The number of $1 \mathrm{~s}$ forming the cell decides the threshold for a cell to hold together

\}

Determination of constituents (Implicants, " $I$ ") of each of the cells of "C":

The compositions which contributed to 1 s are determined.

\}

\{

Removal of weaker cells having lower strengths of adhesion-Phenotypic fitness

Cells with weaker cells die out.

Removal continues till distinct and fitter cells remain.

\}

\}

\}

\}

\{

Cycle II: Cell Grouping

Distinct cells group themselves based on the fitness function defined.

Grouping continues till objective is met.

\}

Algorithm 1: Womb for combinatorial circuits development. 
3.6. Mapping. The proposed design is aimed at generating the net-list of the design. The genetic strip given in the example-100100111011011010111-producing $P \cdot Q$ - maps the interpreted function into a net-list as $Y \leq P$ and $Q$. The netlist generated could be validated using a reconfigurable device, and the same could be fed to the back end tool for implementation. The development time taken by a design is determined by the complexity of the circuit being developed in analogous to the biological world where embryonic development among different species differs considerably.

\section{Development of Combinational Circuits}

4.1. Two-Input Majority Function. An example of input majority function is taken to illustrate the development. The truth table of a two-input majority function is shown in Table 6.

4.2. Length of the Genetic Strip. The length of the strip is defined from equation (1) which makes it 21 bits.

4.3. Cycle I. The inputs considered are level I (primary) inputs. The circuit level considered is level I as in the case of any Boolean SAT problem.

\begin{tabular}{|c|c|c|c|c|c|c|}
\hline Telomere & A & B & $\mathrm{C}$ & $\mathrm{D}$ & $\mathrm{E}$ & Telomere \\
\hline \multirow[b]{2}{*}{1} & 001 & 001 & \multirow[b]{2}{*}{$\begin{array}{l}\text { Not yet } \\
\text { defined }\end{array}$} & \multirow[b]{2}{*}{$\begin{array}{l}\text { Not yet } \\
\text { defined }\end{array}$} & \multirow{2}{*}{$\begin{array}{l}\text { Not yet } \\
\text { defined }\end{array}$} & \multirow[b]{2}{*}{1} \\
\hline & $\begin{array}{c}\text { Primary } \\
\text { inputs }\end{array}$ & $\begin{array}{c}\text { Circuit } \\
\text { level I }\end{array}$ & & & & \\
\hline
\end{tabular}

The problem in hand dictates the choice of gates chosen initially. Since the problem is a Boolean SAT problem, the chosen gate is an AND gate.

\begin{tabular}{|c|c|c|c|c|c|c|}
\hline Telomere & A & B & $\mathrm{C}$ & $\mathrm{D}$ & $\mathrm{E}$ & Telomere \\
\hline \multirow[b]{2}{*}{1} & 001 & 001 & \multirow[b]{2}{*}{$\begin{array}{l}\text { Not yet } \\
\text { defined }\end{array}$} & \multirow[b]{2}{*}{$\begin{array}{l}\text { Not yet } \\
\text { defined }\end{array}$} & $01-011$ & \multirow[b]{2}{*}{1} \\
\hline & $\begin{array}{c}\text { Primary } \\
\text { inputs }\end{array}$ & $\begin{array}{r}\text { Circuit } \\
\text { level I }\end{array}$ & & & $\begin{array}{l}\text { Index is } \\
1 \text {, and the } \\
\text { gate is } \\
\text { AND } \\
\text { gate }\end{array}$ & \\
\hline
\end{tabular}

4.4. Constraints. Constraint 1 -number of inputs $=3$; constraint 2 -final cell population $=4$; constraint 3 - minterms in the truth table which have logic " $1 "=\left[\begin{array}{llll}3 & 5 & 6 & 7\end{array}\right]$.

\begin{tabular}{|c|c|c|c|c|c|c|}
\hline Telomere & A & B & C & $\mathrm{D}$ & $\mathrm{E}$ & Telomere \\
\hline & 001 & 001 & $11-111$ & 011 & $01-011$ & \multirow[b]{2}{*}{1} \\
\hline 1 & $\begin{array}{c}\text { Primary } \\
\text { inputs }\end{array}$ & $\begin{array}{c}\text { Circuit } \\
\text { level I }\end{array}$ & $\begin{array}{l}3 \text { inputs } \\
\text { and all } 3 \\
\text { are taken }\end{array}$ & $\begin{array}{c}1^{\text {st }} 2 \\
\text { inputs } \\
\text { straight } \\
\text { and } 3^{\text {rd }} \\
\text { comple } \\
\text { mented }\end{array}$ & $\begin{array}{l}\text { Index } \\
\text { is } 1, \\
\text { and the } \\
\text { gate is } \\
\text { AND } \\
\text { gate }\end{array}$ & \\
\hline
\end{tabular}

TABLE 6: Truth table of 2-input majority function.

\begin{tabular}{llll}
\hline$P$ & $Q$ & $R$ & $Z$ \\
\hline 0 & 0 & 0 & 0 \\
0 & 0 & 1 & 0 \\
0 & 1 & 0 & 0 \\
0 & 1 & 1 & 1 \\
1 & 0 & 0 & 0 \\
1 & 0 & 1 & 1 \\
1 & 1 & 0 & 1 \\
1 & 1 & 1 & 1 \\
\hline
\end{tabular}

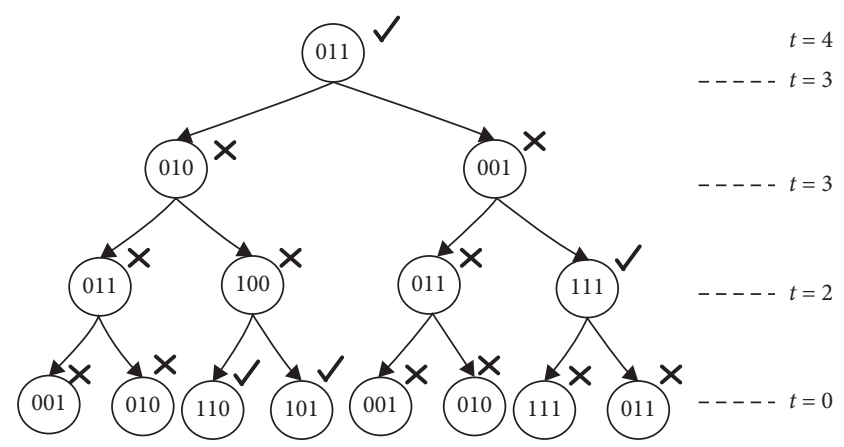

Figure 4: Cell division for a 2-input majority function with 3 input variables.

TABLE 7: Cells after cell division.

\begin{tabular}{lcc}
\hline Cell number & Cells & Minterm \\
\hline 1 & 100100111111110010111 & $\mathrm{P}^{\prime} \mathrm{QR}$ \\
2 & 100100111111101010111 & $\mathrm{PQ}^{\prime} \mathrm{R}$ \\
3 & 100100111111011010111 & $\mathrm{PQR}^{\prime}$ \\
4 & 100100111111111010111 & $\mathrm{PQR}$ \\
\hline
\end{tabular}

4.5. Phase I: Fertilization. Thus, the fertilized zygote is given by 100100111111011010111 . The genetic strip of the chromosome is tested for its fitness. The content of the $\mathrm{D}$ register is tested for fitness. Since 011 has logical " 1 " in the truth table, it is tested fit.

4.6. Phase II: Cell Division: Embryogenesis-Genotypic Fitness. The telomere index and the final products of the cell division are shown in Figure 4. Each parent cell produces two daughter cells which differ from their parent cell by one bit. The inducing factor is taken from right to left. Only the contents of the D register are shown in Figure 4.

The final cell population is given by $n=4$.

4.7. Phase III: Cell Adhesion-Morphogenesis. There are 4 cells at the end of phase II as given in Table 7; all the 4 cells are tested for their specificity factor (s-factor) with all the other cells. Thus, 4 cells after $n \mathrm{C}_{2}$ result in 6 combinations. The higher s-factor dictates the combinations of cells which adhere themselves. Table 8 shows the cells and their s-factor.

The sorted combinations of cells based on $s$-factor are shown in Table 9. 
TABLE 8: Cells and their s-factor.

\begin{tabular}{|c|c|c|c|}
\hline Cell combinations & Cells & Ex-OR & s-factor \\
\hline \multirow{2}{*}{1 with $2 P^{\prime} Q R$ with $P Q^{\prime} R$} & 100100111111110010111 & 100100111111110010111 & \multirow{2}{*}{0.91} \\
\hline & 100100111111101010111 & 100100111111101010111 & \\
\hline \multirow{2}{*}{1 with $3 P^{\prime} Q R$ with $P Q R^{\prime}$} & 100100111111110010111 & 100100111111110010111 & \multirow{2}{*}{0.91} \\
\hline & 100100111111011010111 & 100100111111011010111 & \\
\hline \multirow{2}{*}{1 with $4 P^{\prime} Q R$ with $P Q R$} & 100100111111110010111 & 100100111111110010111 & \multirow[b]{2}{*}{0.96} \\
\hline & 100100111111111010111 & 100100111111111010111 & \\
\hline \multirow{2}{*}{2 with $3 P Q^{\prime} R$ with $P Q R^{\prime}$} & 100100111111101010111 & 100100111111101010111 & \multirow{2}{*}{0.91} \\
\hline & 100100111111011010111 & 100100111111011010111 & \\
\hline \multirow{2}{*}{2 with $4 P Q^{\prime} R$ with $P Q R$} & 100100111111101010111 & 100100111111101010111 & \multirow{2}{*}{0.96} \\
\hline & 100100111111111010111 & 100100111111111010111 & \\
\hline \multirow{2}{*}{3 with $4 P Q R^{\prime}$ with $P Q R$} & 100100111111011010111 & 100100111111011010111 & \multirow{2}{*}{0.96} \\
\hline & 100100111111111010111 & 100100111111111010111 & \\
\hline
\end{tabular}

TABLE 9: Cells sorted based on their s-factor.

\begin{tabular}{|c|c|c|c|}
\hline Cell combina tions & Cells & Ex-OR & s-factor \\
\hline \multirow{2}{*}{1 with $4 P^{\prime} Q R$ with $P Q R$} & 100100111111110010111 & 100100111111110010111 & \multirow{2}{*}{0.96} \\
\hline & 100100111111111010111 & 100100111111111010111 & \\
\hline \multirow{2}{*}{2 with $4 P Q^{\prime} R$ with $P Q R$} & 100100111111101010111 & 100100111111101010111 & \multirow{2}{*}{0.96} \\
\hline & 100100111111111010111 & 100100111111111010111 & \\
\hline \multirow{2}{*}{3 with $4 P Q R^{\prime}$ with $P Q R$} & 100100111111011010111 & 100100111111011010111 & \multirow{2}{*}{0.96} \\
\hline & 100100111111111010111 & 100100111111111010111 & \\
\hline \multirow{2}{*}{1 with $2 P^{\prime} Q R$ with $P Q^{\prime} R$} & 100100111111110010111 & 100100111111110010111 & \multirow{2}{*}{0.91} \\
\hline & 100100111111101010111 & 100100111111101010111 & \\
\hline \multirow{2}{*}{1 with $3 P^{\prime} Q R$ with $P Q R^{\prime}$} & 100100111111110010111 & 100100111111110010111 & \multirow{2}{*}{0.91} \\
\hline & 100100111111011010111 & 100100111111011010111 & \\
\hline \multirow{2}{*}{2 with $3 P Q^{\prime} R$ with $P Q R^{\prime}$} & 100100111111101010111 & 100100111111101010111 & \multirow{2}{*}{0.91} \\
\hline & 100100111111011010111 & 00100111111011010111 & \\
\hline
\end{tabular}

Table 10: Cells and their $r$-index.

\begin{tabular}{|c|c|c|c|c|c|c|}
\hline \multirow{2}{*}{ Cell adhesion } & \multirow{2}{*}{ Adhesion } & \multirow{2}{*}{ s-factor } & \multicolumn{3}{|c|}{$r$-index } & \\
\hline & & & 1 & 2 & 3 & 4 \\
\hline 1 with $4 P^{\prime} Q R$ with $P Q R$ & $\begin{array}{l}100100111111110010111 \\
100100111111111010111\end{array}$ & 0.96 & 1 & 0 & 0 & 1 \\
\hline 2 with $4 P^{\prime} Q R$ with $P Q R$ & $\begin{array}{l}100100111111101010111 \\
100100111111111010111\end{array}$ & 0.96 & 0 & 1 & 0 & 1 \\
\hline \multirow[t]{2}{*}{3 with $4 P Q R^{\prime}$ with $P Q R$} & $\begin{array}{l}100100111111011010111 \\
100100111111111010111\end{array}$ & 0.96 & 0 & 0 & 1 & 1 \\
\hline & & Total, $r$ & 1 & 1 & 1 & 3 \\
\hline
\end{tabular}

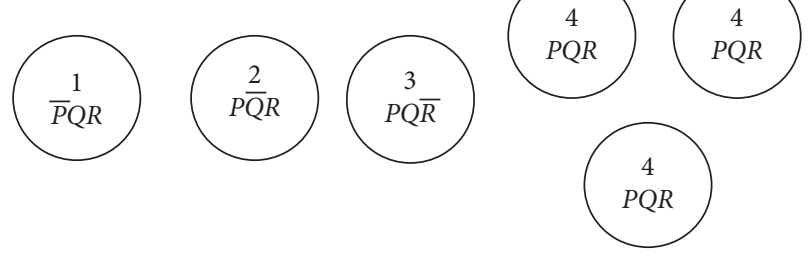

FIgURe 5: Local cell division.

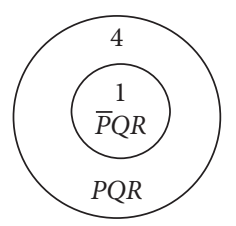

(a)

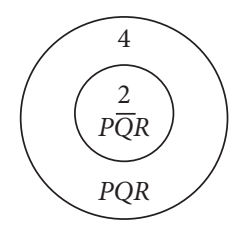

(b)

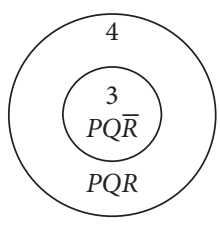

(c)
Figure 6: Cell adhesion.
The $r$-index or the replication index tells how much copies each cell in the combinations makes. For this to happen, the combination must have higher $(\geq 0.95) \mathrm{s}$-factor. Each cell is given $(r-1)$ copies. Table 10 shows the replication index ( $r$-index) of cells after adhesion.
4.8. Local Cell Division. Each cell makes $(r-1)$ copies of it. This is shown in Figure 5.

Each cell adheres to the other cell types. Cells 1-3 combine with the three copies of cell 4 as shown in Figure 6. 
TABLE 11: Gate-dominated adhesions.

\begin{tabular}{lrr}
\hline Cell adhesion & Adhesion & Adhered cell \\
\hline \multirow{2}{*}{ G1 $4 P^{\prime} Q R$ G1 $P Q R$} & $\mathbf{1 0 0 1 0 0 1 1 1 1 1 1 1 0 0 1 0 1 1 1}$ & $\mathbf{1 0 0 1 0 0 1 1 1 1 1 1 1 1 0 0 1 0 1 1 1}$ \\
& 10010011111111010111 & $\mathbf{1 0 0 1 0 0 1 1 1 1 1 1 0 1 0 1 0 1 1 1}$ \\
G $14 P^{\prime}$ G G1 $P Q R$ & $\mathbf{1 0 0 1 0 0 1 1 1 1 1 1 0 1 0 1 0 1 1 1}$ & $\mathbf{1 0 0 1 0 0 1 1 1 1 1 0 1 1 0 1 0 1 1 1}$ \\
\hline
\end{tabular}

TABLE 12: Layered mutations.

\begin{tabular}{lccc}
\hline Cell & Cell adhesion & Adhered cell & New adhered cell after layered mutation \\
\hline 1 & $Q R$ & $\mathbf{1 0 0 1 0 0 1 1 1 1 1 1 1 1 0 0 1 0 1 1 1}$ & $\mathbf{1 0 0 1 0 0 1 1 1 1 1 0 1 1 0 0 1 0 1 1 1}$ \\
2 & $P R$ & $\mathbf{1 0 0 1 0 0 1 1 1 1 1 1 1 0 1 0 1 0 1 1 1}$ & $\mathbf{1 0 0 1 0 0 1 1 1 1 0 1 1 0 1 0 1 0 1 1 1}$ \\
3 & $P Q$ & $\mathbf{1 0 0 1 0 0 1 1 1 1 1 1 0 1 1 0 1 0 1 1 1}$ & $\mathbf{1 0 0 1 0 0 1 1 1 0 1 1 0 1 1 0 1 0 1 1 1}$ \\
\hline
\end{tabular}

TABLe 13: Cells and their s-factors.

\begin{tabular}{lcccc}
\hline Cell & Cell adhesion & Cell combinations & Ex-OR & s-factor \\
\hline 1 & QR & 1 with 2 QR with PR & 100100111110110010111 & 100100111101101010111 \\
2 & $P R$ & 1 with 3 QR with PQ & 100100111110110010111 & $\mathbf{0 . 8 3}$ \\
3 & & & 100100111011011010111 & $\mathbf{0 . 9 2}$ \\
& & 2 with 3 PR with PQ & 100100111101101010111 & $\mathbf{0 . 8 3}$ \\
\hline
\end{tabular}

Gate-dominated adhesions, gadhrines (gadherin type $1-\mathrm{GT} 1$ ), are given in Table 11 .

4.9. Layered Mutation. Layered mutation will affect a corresponding change in the $\mathrm{C}$ frame, i.e., a second bit change in the $\mathrm{D}$ frame due to cell adhesion will cause a corresponding second bit change in the $\mathrm{C}$ frame.

The new fused cell has a bit changed to logic ' 0 ' in frame C corresponding to the allele which is fused (adhered) in frame D. Table 12 shows the layered mutation.

4.10. Cell Adhesions. There are 3 cells at the end of first local cell division, and all the 3 cells are tested for their specificity factor (s-factor) with all the other cells as given in Table 13. Thus, 3 cells after $n \mathrm{C}_{2}$ result in 3 combinations.

The higher s-factor $(\leq 0.95)$ dictates the combinations of cells which adhere themselves.

No combination has high s-factor. Hence, cells have $r$-index as numerical 0 , which means no replication and no further adhesion. All the three cells are distinct as shown in Table 14.

4.11. Differential Strength of Adhesion. The differential strength of adhesion between cells is determined to find out the redundant cells forming the organism. The contents of frames $\mathrm{C}$ and $\mathrm{D}$ determine the differential strength of adhesion.

4.12. Determination of the Number of 1 s Forming Each Cell. The last column of Table15indicates the number of $1 \mathrm{~s}$ forming the cell. The difference between the number of
TABle 14: Cells after cell adhesion.

\begin{tabular}{lcc}
\hline Cell & Cell adhesion & Cell \\
\hline 1 & QR & 100100111110110010111 \\
2 & $P R$ & 100100111101101010111 \\
3 & $P Q$ & 100100111011011010111 \\
\hline
\end{tabular}

inputs and the number of inputs grouped eventually is used to determine the number of $1 \mathrm{~s}$ in a cell.

Table 16 shows that there are two $1 \mathrm{~s}$ in each of the cells. The last column in Table 16 indicates the number of $1 \mathrm{~s}$. All the cells- $Q R, P R$, and $P Q$-look identical as far as the number of $1 \mathrm{~s}$ making up the group is considered. Figure 7 testifies Table 16 with the help of a Karnaugh map.

The Ex-OR operation of the D frame and c2 subframe results in $0 \mathrm{~s}$. This indicates that there are no changes already done to the $\mathrm{D}$ frame as given in Table 17. The presence of logical 0 after the Ex-OR of the c2 subframe and D frame indicates that $0 \mathrm{~s}$ in the product can be toggled to $1 \mathrm{~s}$ to determine the constituents (implicants) of the cells. In contrast, the presence of logical 1 after the Ex-OR of the c2 subframe and $\mathrm{D}$ frame indicates that 0 s in the corresponding allele should not be toggled to find out the constituents (implicants) of the cell.

4.13. Removal of Weaker Cells Having Lower Strengths of Adhesion: Phenotypic Fitness. To remove the weaker constituent from the group of cells, the individual strength of each of the constituent is checked. Section 3 describes in detail the idea behind the differential strength of adhesion. Each of the constituent in the present example takes a 
TABle 15: Determination of $1 \mathrm{~s}$ in each cell.

\begin{tabular}{|c|c|c|c|c|c|c|}
\hline \multirow{3}{*}{ Cell } & \multirow{3}{*}{ Cell } & \multicolumn{4}{|c|}{ C frame interpretation } & \multirow{3}{*}{ No. of $1 \mathrm{~s}$ in the cell, $f=2^{\mathrm{cl}-\mathrm{c} 2}$} \\
\hline & & \multicolumn{2}{|c|}{$\mathrm{cl}$} & \multicolumn{2}{|r|}{ c2 } & \\
\hline & & Encoded & No. of inputs & String & Inputs considered & \\
\hline 1 & $Q R$ & 11 & 3 & 110 & 2 & 2 \\
\hline 2 & $P R$ & 11 & 3 & 101 & 2 & 2 \\
\hline 3 & $P Q$ & 11 & 3 & 011 & 2 & 2 \\
\hline
\end{tabular}

TABle 16: Differential strength of adhesion.

\begin{tabular}{lccccc}
\hline Cell & Cell & Cell & & C frame & c2 \\
\hline 1 & $Q R$ & 100100111110110010111 & c1 & 11 & 110 \\
2 & $P R$ & 100100111101101010111 & 11 & 101 & 110 \\
3 & $P Q$ & 100100111011011010111 & 11 & 011 & 011 \\
\hline
\end{tabular}

\begin{tabular}{|c|c|c|c|c|}
\hline & 00 & 01 & 11 & 10 \\
\hline 0 & $0^{0}$ & $0^{1}$ & $1^{3}$ & $0^{2}$ \\
\hline 1 & $0^{4}$ & & $1^{7}$ & $\begin{array}{r}6 \\
1\end{array}$ \\
\hline
\end{tabular}

(a)

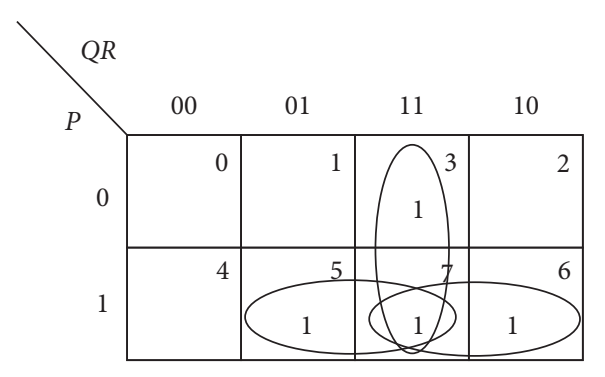

(b)

Figure 7: Differential strength of adhesion. (a) $[3,5,6,7]$. (b) Group $(3,7),(6,7),(5,7)$.

TABle 17: Determination of the constituent of each cell.

\begin{tabular}{|c|c|c|c|c|c|c|c|c|}
\hline \multirow{3}{*}{ Cell } & \multicolumn{3}{|c|}{ C frame } & & \multicolumn{4}{|c|}{ D frame interpretation } \\
\hline & Cell & \multirow{2}{*}{$\mathrm{c} 1$} & \multirow{2}{*}{$\mathrm{c} 2$} & \multirow[t]{2}{*}{$\mathrm{D}$ frame (3 inputs) } & \multirow{2}{*}{ c2 XOR D } & \multirow{2}{*}{ Change already } & \multicolumn{2}{|c|}{ D frame } \\
\hline & & & & & & & Encoded & Actual \\
\hline \multirow{2}{*}{1} & \multirow{2}{*}{$Q R$} & \multirow{2}{*}{11} & \multirow{2}{*}{110} & \multirow{2}{*}{110} & \multirow{2}{*}{000} & \multirow{2}{*}{ No bit } & 110 & 3 \\
\hline & & & & & & & 111 & 7 \\
\hline \multirow{2}{*}{2} & \multirow{2}{*}{$P R$} & \multirow{2}{*}{11} & \multirow{2}{*}{101} & \multirow{2}{*}{101} & \multirow{2}{*}{000} & \multirow{2}{*}{ No bit } & 101 & 5 \\
\hline & & & & & & & 111 & 7 \\
\hline \multirow[t]{2}{*}{3} & \multirow[t]{2}{*}{$P Q$} & \multirow[t]{2}{*}{11} & \multirow{2}{*}{011} & \multirow{2}{*}{011} & \multirow{2}{*}{000} & \multirow{2}{*}{ No bit } & 011 & 6 \\
\hline & & & & & & & 111 & 7 \\
\hline
\end{tabular}

TABLE 18: Constituents (implicants) of the cell.

\begin{tabular}{lllr}
\hline Cell & & Threshold & \\
& $F$ & $1 / f$ & Constituents \\
\hline$Q R$ & 2 & 0.5 & $(3,7)$ \\
$P R$ & 2 & 0.5 & $(5,7)$ \\
$P Q$ & 2 & 0.5 & $(6,7)$ \\
\hline
\end{tabular}

"strength" of 0.5 since there are two constituents in each cell. Table 18 shows the individual "strength."

Each adhesion makes the individual constituent to lose half of its strength to the fellow constituent. In Table 19, constituent 7 in the cell $(3,7)$ has a "strength" of 0.5 after adhesion 1 . The same constituent loses half of its "strength" and becomes 0.25 as it undergoes one more adhesion. The individual constituent or implicant can survive to make as many adhesions as possible with the condition that the combined strength of adhesion of the individual cell should not fall below the initial strength as given in Table 18. 
TABle 19: Determination of strengths of individual cells.

\begin{tabular}{lcccccccccccc}
\hline \multirow{2}{*}{ Constituents } & \multicolumn{3}{c}{ Adhesion 1} & \multicolumn{4}{c}{ Adhesion 2} \\
& 3 & 5 & 6 & 7 & 3 & 5 & 6 & 7 & 3 & 5 & 6 \\
\hline$Q R(3,7)$ & 0.5 & 0 & 0 & 0.5 & 0.5 & 0 & 0 & 0.25 & 0.5 & 0 & 0 \\
$P R(5,7)$ & - & - & - & - & 0 & 0.5 & 0 & 0.25 & 0 & 0.5 & 0 & 0.25 \\
$P Q(6,7)$ & - & - & - & - & - & - & - & - & 0 & 0 & 0.5 & 0.125 \\
\hline
\end{tabular}

TABle 20: Differential strength of adhesion.

\begin{tabular}{|c|c|c|c|c|c|c|}
\hline \multirow{2}{*}{ Constituents } & \multicolumn{4}{|c|}{ Adhesion 3} & \multirow{2}{*}{ Total } & \multirow{2}{*}{ Strengths of adhesion } \\
\hline & 3 & 5 & 6 & 7 & & \\
\hline$Q R(3,7)$ & 0.5 & 0 & 0 & 0.25 & 0.75 & Strong \\
\hline$P R(5,7)$ & 0 & 0.5 & 0 & 0.125 & 0.625 & Strong \\
\hline$P Q(6,7)$ & 0 & 0 & 0.5 & 0.125 & 0.625 & Strong \\
\hline
\end{tabular}

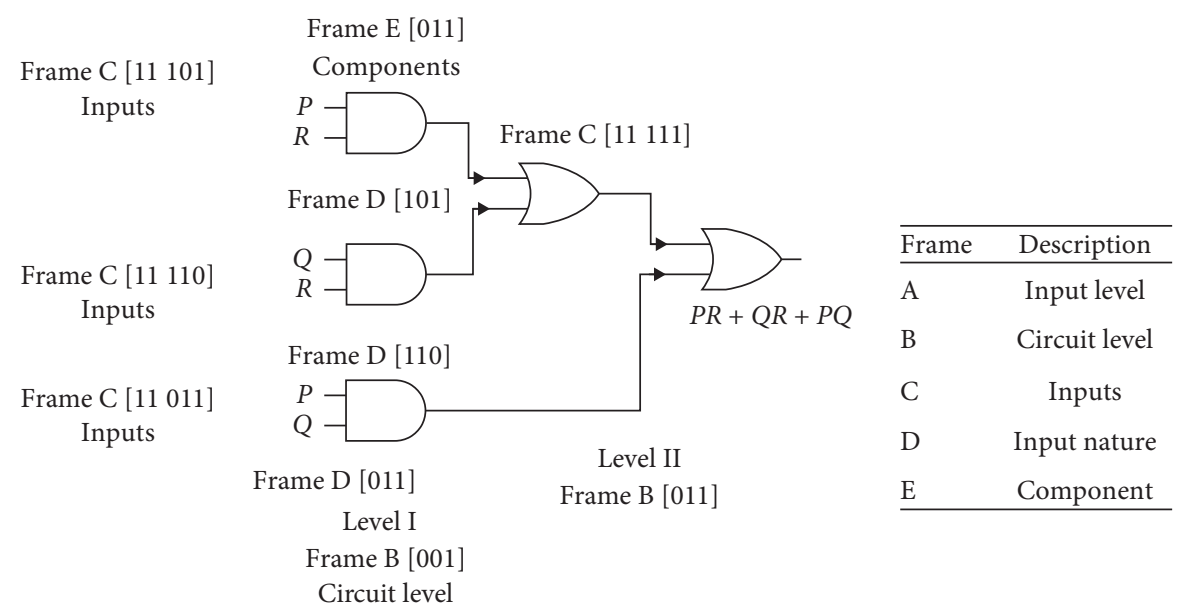

FIGURE 8: Implementation of 2-input majority function with 3 inputs.

TABle 21: Combinational circuits and their genomes.

\begin{tabular}{lcccc}
\hline Circuits & Inputs & Length of the cell (bits) & No. of cells & Length of the genome (bits) \\
\hline 2-input Ex-OR gate & 2 & 18 & 3 & $3 \times 18$ \\
2-input majority function & 3 & 21 & 4 & $4 \times 21$ \\
2-input majority function & 4 & 23 & 10 & $10 \times 23$ \\
2-to-1 multiplexer circuit & 3 & 21 & 3 & $3 \times 21$ \\
\hline
\end{tabular}

All the three cells do have higher strength of adhesion, hence forms the cells in cycle I of development as shown in Table 20.

4.14. Cycle II: Cell Grouping. Cycle II of the development has minor changes from the previous cycle. Input level and circuit level are updated in frames $\mathrm{A}$ and $\mathrm{B}$, respectively. Frames $\mathrm{C}$ and $\mathrm{D}$ have the inputs decoded and are taken straight. The outputs of cycle I form the inputs for cycle II. OR gate forms the component of interest in cycle II as the problem in hand is a Boolean SAT problem. The number of inputs is 3 (more than 2 and odd) at the end of cycle I. The 3 inputs are grouped into 2, and the output of the OR gate is further made to undergo logical OR operation with the third input to ensure two-input gate implementation. The circuit level is interpreted as the same for all the three inputs, i.e., the 3-input OR gate is made up of two 2-input OR gates.

The following cells constitute the 2-input majority function:

$$
\begin{aligned}
& 100100111110110010111 \\
& 100100111101101010111 \\
& 100100111011011010111 \\
& 101101111111111010101
\end{aligned}
$$

The same can be interpreted as follows. Decoding a 2input majority function with 3 inputs results in a genetic strip of $4 \times 21$ bits given by 100100111110110010 111100100111101101010111100100111011011010111011011 
11111111010101. Figure 8 shows the implementation of 2-input majority function with each frame explicitly shown.

\section{Developmental Results}

Application of the proposed algorithm to three primitive combinatorial circuits gave the following results as listed in Table 21. The length of the genome increases with the increase in the number of inputs. The total number of inputs determines the cell length, and the number of logical $1 \mathrm{~s}$ in the truth table determines the number of cells which in turn determines the length of the entire genome.

Table 21, the length of the cell and the eventual length of the genome depend on the type of the circuit being solved. This is analogous to the biological world in which each species' genome is unique. However, there are certain similarities in the genome of various species. This is true to the genome of different circuits as well as illustrated earlier.

\section{Conclusion}

The development of a combinational digital circuit based on developmental biology is carried out for 4 input circuits. The development of a digital cirucit from a single cell is seen to follow similar steps as in the natural world. The development is conceptualized around a 23 bit binary string for the primitive 4 input circuits. The length of the genome increases with the increase in the number of input bit width. For a circuit with a maximum of 8 bit input, the length of the genetic strip is 32 bits. For a 16 bit input, the length of the genome will be 49 bit length. The development of combinational circuits can be extended up to 7 levels with the empirical relationship presented in this paper. For bits of higher order, say 128 bits, the length of the genome increases proportionately. For instance, the length of a single cell would be 155 bits. The development of higher-order strings would be taken up as the future work. As an illustration, primitive combinational circuits such as 2-input Ex-OR gate, 2input majority function for a 3-input circuit, and a 2-to-1 multiplexer circuit are taken up for the developmental algorithm developed. The examples show how the circuit develops in the presence of redundant terms. The future work would consider implanting the same with a sophisticated tool to perform the generation and mapping of the particular evolved net-lists. However, the present work ends with mapping of the strips to the specified components using the simulation tool.

\section{Data Availability}

No Data were used to support this study. However, the findings can be used by researchers for their future work.

\section{Conflicts of Interest}

The authors declare that they have no conflicts of interest.

\section{References}

[1] B. Xin, J. Chen, Z. Peng, and F. Pan, "An adaptive hybrid optimizer based on particle swarm and differential evolution for global optimization," Science China Information Sciences, vol. 53, no. 5, pp. 980-989, 2010.

[2] J. D. Lohn, "Evolutionary design of a single-wire circularlypolarised X-band antenna for NASA's space technology 5 mission," in Proceedings of the 2005 IEEE Antennas and Propagation Society International Symposium, pp. 267-270, Washington, DC, USA, 2005.

[3] P. Bentley and J. Wakefield, "The table: an illustration of evolutionary design using genetic algorithms," in Proceedings of the First International Conference on Genetic Algorithm in Engineering Systems: Innovations and Applications, pp. 412418, 1995.

[4] R. J. Preen and L. Bull, "Toward the coevolution of novel vertical-axis wind turbines," IEEE Transactions on Evolutionary Computation, vol. 19, no. 2, pp. 284-294, 2015.

[5] A. Thompson, "Silicon evolution," in Proceedings of Genetic Programming 1996 (GP96), J. R. Koza, Ed., pp. 444-452, MIT Press, Cambridge, MA, USA, 1996.

[6] Y. del Valle, G. K. Venayagamoorthy, S. Mohagheghi, J.-C. Hernandez, and R. G. Harley, "Particle swarm optimization: basic concepts, variants and applications in power systems," IEEE Transactions on Evolutionary Computation, vol. 12, no. 2, pp. 171-195, 2008.

[7] B. Wang, X. Jin, and B. Cheng, "Lion pride optimizer: an optimization algorithm inspired by lion pride behavior," Science China Information Sciences, vol. 55, no. 10, pp. 2369-2389, 2012.

[8] B. R. Rajakumar, “The lion's algorithm: a new nature-inspired search algorithm," Procedia Technology, vol. 6, pp. 126-135, 2012.

[9] B. R. Rajakumar, "Lion algorithm for standard and large scale bilinear system identification: a global optimization based on lion's social behavior," in Proceedings of the 2014 IEEE Congress on Evolutionary Computation (CEC), July 2014, Beijing, China.

[10] T. Ray and K. M. Liew, "Society and civilization: an optimization algorithm based on the simulation of social behavior," In IEEE Transactions on Evolutionary Computation, vol. 7, no. 4, 2003.

[11] W. Pedrycz, G. Succi, and O. Shai, "Genetic-fuzzy approach to the boolean satisfiablity problem," Letters in IEEE Transactions on Evolutionary Computation, vol. 6, no. 5, 2002.

[12] K. A. De Jong and W. M. Spears, "Using genetic algorithms to solve NP- complete problems," in Proceedings of the $3 \mathrm{rd}$ International Conference on Genetic Algorithms, pp. 124-132.

[13] A. Slowik and M. Bialko, "Evolutionary design of combinational digital circuits: state of the art, main problems and future trends," in Proceedings of the 1st International Conference on Information Technology, May 2008, Gdansk, Poland.

[14] C. MacLeod, An Introduction to Practical Neural Networks and Genetic Algorithms for Engineers and Scientists, The Robert Gorden University, Aberdeen, UK, 2010.

[15] J. Koza, Genetic Programming, on the Programming of Computers by Means of Natural Selection, The MIT Press, Cambridge, MA, USA, 1992.

[16] C. A. C. Coello, "Using genetic algorithms to design combinational circuits," Intelligent Engineering Through Artificial Neural Networks, vol. 6, pp. 391-396, 1996.

[17] J. F. Miller, P. Thomson, and T. Fogarty, "Designing electronic circuits using evolutionary algorithms, arithmatic circuits: a case study," Genetic Algorithms and Evolution Strategy in Engineering and Computer Science, pp. 105-131, John Wiley \& Sons, Inc., Chichester, UK, 1997. 
[18] T. Kalganova, J. F. Miller, and T. C. Fogarty, "Some aspects of the evolvable hardware for multiple-valued combinational circuit design," Evolvable Systems: From Biology to Hardware, pp. 78-89, Lausanne, Switzerland, 1998.

[19] J. F. Miller, T. Kalganova, N. Lipintskaya, and D. Job, “The genetic algorithm as a discovery engine: strange circuits and new principles," in Proceedings of the AISB Symposium on Creative Evolutionary Systems (CES '99), Edinburgh, UK, 1999.

[20] T. Gordon and P. Bentley, "Development Brings Scalability to Hardware Evolution," in Proceedings of the 2005 NASA/DoD Conference on Evolvable Hardware (EH05), pp. 272-279, Washington, DC, USA, 2005.

[21] J. M. W. Slack, Essential Developmental Biology, John Wiley \& Sons, Ltd., Chichester, UK, 2nd edition, 2013. 